\title{
An algorithm for responsive fluid resuscitation at the Western Cape Provincial Tertiary Adult Burn Centre
}

W G Kleintjes, ${ }^{1}$ MB ChB, MMed (Plast), FC (Plast Surg) SA, PhD; C A Baars, ${ }^{2}$ MB BCh; H Parker, ${ }^{2}$ MB ChB; C Augustynn, ${ }^{2}$ MB ChB; W Grove, ${ }^{2}$ MB ChB; E P Kotzee, ${ }^{2} \mathrm{MB} C \mathrm{ChB}$

\author{
Western Cape Provincial Tertiary Adult Burn Unit, Division of Surgery, Tygerberg Hospital and Stellenbosch University, South Africa \\ 2Division of Surgery, Tygerberg Hospital, Cape Town, South Africa
}

Corresponding author: C A Baars (chiaraanne@gmail.com)

\begin{abstract}
Fluid resuscitation and its role in the management of burns patients has an important link to the outcome of these patients and their injuries. The algorithm designed in this research utilises the modified Parklands formula, and includes instances where albumin can limit fluid overload in severe cases that may require large volumes of fluid resuscitation. It is also a schematic intended to be understandable and easy to execute by various staff members, as part of the resuscitation team. As such, it is not all-inclusive, but covers the important areas of fluid balance, and additional measurements to complete the resuscitation process in qualifying patients with burn injuries.
\end{abstract}

SAfr J Plast Reconstr Aesthet Burns Surg 2018;1(1):16-18. DOI: 10.7196/SAJPRASB.2018.v1i1.5

Fluid resuscitation is paramount in burn injuries, as it is one major foothold by which outcomes and morbidity can be determined. ${ }^{[1]}$ This pivotal skill, which has not yet been perfected, has proven to be complex in its significance and influence. There are numerous variations on formulas that have been developed in an attempt to determine the ideal fluid resuscitation process in burns patients, but the development of an inclusive algorithm that utilises a resuscitation formula as well as other components of holistic management is the purpose of this review. ${ }^{[1-3]}$

The inclusion of the albumin protocol within this algorithm serves as an important intervention in patients who require massive volumes of initial resuscitation, in order to reduce the total fluid requirement and subsequent overloaded states, while not significantly worsening the burn injury. ${ }^{[3]}$

Modern trends in fluid resuscitation have been to use less aggressive fluid resuscitation regimens, to avoid complications of over-resuscitation. ${ }^{[4]}$

Despite having protocols adopted from other units, we found it difficult to teach staff the application of the protocols. We decided to develop an algorithm for fluid resuscitation based on the University of Utah algorithm, ${ }^{[5]}$ in the hope that it would assist staff with practical adjustments needed during fluid resuscitation. Our algorithm is illustrated in Fig. 1.

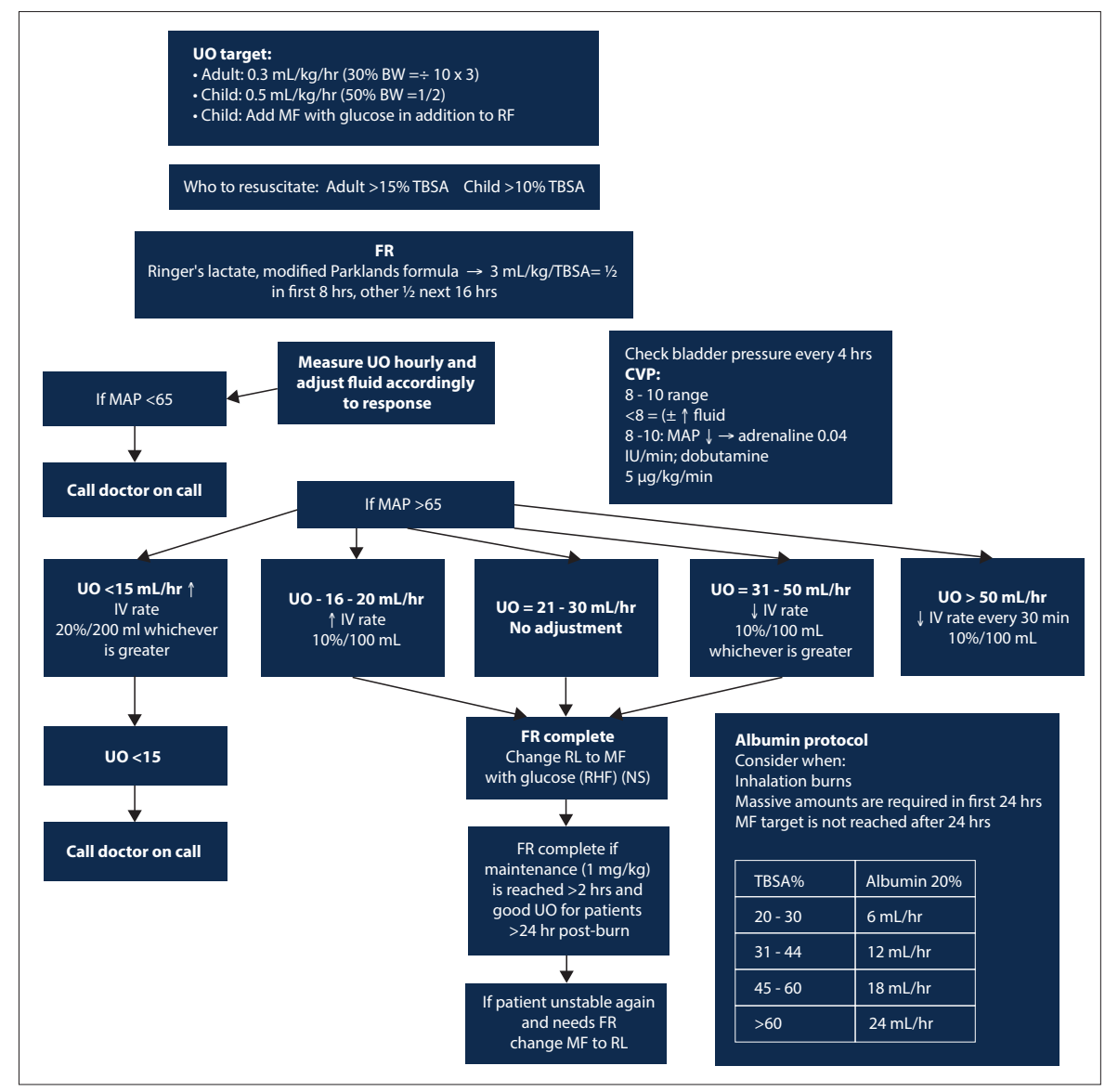

Fig. 1. The burn resuscitation algorithm used in our centre. ( $U O=$ urine output; $B W=$ bodyweight; $M F=$ maintenance fluid; $F R=$ fluid resuscitation; $T B S A=$ total body surface area; $I V=$ intravenous; $C V P=$ central venous pressure; $M A P=$ mean arterial pressure; $R L=$ Ringer's lactate; $R H F=$ rehydration fluid; $N S=$ normal saline.) 


\section{Discussion}

Urine output $(\mathrm{UO})$ is the most important clinical parameter that we use to assess the adequacy of our resuscitation in terms of the goal organ perfusion. ${ }^{[5,6]}$ Modern trends have been to target lower urine output volumes than those given in standard textbooks. ${ }^{\left[{ }^{[4]}\right.}$ For children, UO of 0.5 $\mathrm{mL} / \mathrm{kg} / \mathrm{h}$ is adequate, and for adults we use $0.3 \mathrm{~mL} / \mathrm{kg} / \mathrm{h}$. This is relatively easy to convey to staff; for example, for children we use half the body weight $(0.5=50 \%)$ in $\mathrm{kg}$, in $\mathrm{mL} / \mathrm{h}$, as our target $\mathrm{UO}$, and for adults we divide the body weight (in $\mathrm{kg}$ ) by $10(0.1=10 \%)$, and multiply it by 3 , to get to $30 \%$ (0.3) of the body weight to get the target $U O \mathrm{in} \mathrm{mL} / \mathrm{kg} / \mathrm{h}$. Some authors use a weight of $30 \mathrm{~kg}$ for children, to differentiate a $\cup O$ target of $1 \mathrm{~mL} / \mathrm{kg} / \mathrm{h}$ below $30 \mathrm{~kg}$, and $0.5 \mathrm{~mL} / \mathrm{kg} / \mathrm{h}$ above $30 \mathrm{~kg} .{ }^{[4]}$

It is very important to know who to fluid-resuscitate and who not. One would hope that the inexperienced would be familiar with accurate methods of calculating the total body surface area (TBSA) for fluid resuscitation purposes; alternatively, pictures can be sent by telelink (e.g. the Vula Medical Referral app (Vula Mobile, South Africa)), where the size of burn can be assessed by a burns doctor. We promote using the palmar $1 \%$ rule for accuracy, since we have many patients with variable body size distributions, where a fixed number, as in the rule of nines, has higher risks for inaccuracies. By excluding superficial burns (such as sunburns, where the epidermis is intact and fluid is not lost) and including $10 \%$ for where an inhalation burn is suspected (fluid is lost in the lung parenchyma), the accuracy of the TBSA for fluid resuscitation is increased. This is the provincial burn protocol, and may vary slightly at different facilities. We promote the initiation of fluid resuscitation for children with $>10 \%$, and adults $>15 \%$, TBSA burns. Because children have very low glucose reserves, they should always receive maintenance fluid (MF) (which contains glucose) in addition to the resuscitation fluid (Ringer's lactate $(R L))$ that can be calculated using the 4:2:1 rule.

The Western Cape provincial fluid resuscitation guidelines propose the use of the modified Parklands formula, starting at $3 \mathrm{~mL} / \mathrm{kg} / \mathrm{TBSA}$ for calculating the estimated fluid required for the first 24 hours post burn. According to traditional teaching, half of this volume is given in the first 8 hours after the burn, and the other half in the next 16 hours. This is probably the biggest cause of fluid resuscitation problems we have in burn resuscitation teaching, and in translating knowledge into practice. It is not uncommon that these guidelines are prescribed by a clinician, and the nursing staff are expected to comply without any specific empowerment, and with strict orders to adjust the fluid rates according to the patient's response. Simple terminology might assist staff to grasp the concept of what needs to be achieved. A word such as 'responsive' indicates that action needs to be taken, and it is more instructive in the method of getting to the end point than the term 'goal-directed' fluid resuscitation. Goal-directed refers to an end point that you want to reach, and responsive resuscitation implies active adjustments according to the patient's response ${ }^{[6]}$ A combination of these words exemplifies what we try to achieve in modern burn fluid resuscitation, namely responsive goaldirected fluid resuscitation.

For the sake of simplicity, we chose only to use the mean arterial pressure (MAP) in the algorithm to assist with major judgement calls about the adequacy of the fluid resuscitation. Is it necessary to use inotropes or not? If this question is asked because the MAP dropped to $<65$, a doctor should be alerted, and assist the nursing staff with the resuscitation. It is important to consider the associated parameters, such as heart rate and oxygenation, as well. Other parameters are also not included that would add complexity - such as stroke volume variations and pulse pressure variations, or transpulmonary thermodilution and lactate levels. ${ }^{[7,8]}$ In centres where non-invasive cardiac monitoring is used often and staff are well trained, these parameters can be added to such an algorithm, as even with a relatively simple protocol being available in the burn centre, this does not necessarily translate into the accurate implementation of these guidelines.

Even though we have included central venous pressure (CVP) measurements in the protocol, they are often not measured by our staff. Usually, if the fluid resuscitation is still inadequate, the intensive care unit (ICU) nurses are asked to take serial measurements in order to see the trends, which, in conjunction with other parameters, can be useful in the resuscitation process. Passive leg-raise tests are fairly accurate in predicting fluid responsiveness of patients in cases of uncertainty. Non-invasive cardiac monitoring can give some comfort in situations where it sometimes becomes very difficult to decide what fluid management strategy to follow in the face of multiorgan failure. ${ }^{[9]}$

The regular measurements of bladder pressure are important for large burns, and where large fluid volumes are required for resuscitation, to assess for abdominal compartment syndrome, which is often fatal for these patients. ${ }^{[10]}$ Assessing this for all burn patients admitted to the ICU is definitely not necessary, even though some ICUs adopt this as a routine vital sign. If such tests are not conducted regularly, however, this results in a shortage of junior staff and nurses with the skill to perform them. These are necessary skills and are warranted for suitable patients, where this may result in life-saving interventions.

Even though we advocate that fluid be decreased if the UO target is in the $30-50 \mathrm{~mL} / \mathrm{h}$ range, we often have heavy patients, where this is more or less the target range, and therefore make no RL adjustments. For patients with inhalation burns, we prefer to have the lungs dry rather than wet, and will restrict fluid until we have a slight increase in the serum urea level (in the range of $6-8 \mathrm{mg} / \mathrm{dL}$ ). For inhalation burns or where maintenance levels $(1 \mathrm{~mL} / \mathrm{kg} / \mathrm{h})$ are not reached at 24 hours post burn and resuscitation, we use $20 \%$ albumin if there is a positive response to its initiation (rise in blood pressure and increased UO within 14 minutes), and use furosemide at $1 \mathrm{~mL} / \mathrm{kg}$ 12-hourly, 30 minutes after starting albumin, to further mobilise fluid from the lung and out of the intravascular space. Manipulating fluid from the lungs using the acronym PAL (i.e. PEEP (positive end-expiratory pressure), albumin, furosemide) was promoted by Prof. Manu Malbrein at a South African Burns Society conference in 2013. ${ }^{[10]}$ Apart from using albumin for fluid resuscitation after the first 24 hours and for manipulating fluid shifts in the lung in the acute burned patient, it can also be used later to manipulate limb and organ fluid shifts, in combination with furosemide, to assist with wound healing and avoid other complications.

The 20\% albumin regimen that we use was initially proposed by Bacomo and Chung in 2011. ${ }^{[3]}$ Since its introduction over the last 6 years we have noted more rapid return to MF levels, and patient outcomes have dramatically improved. The contraindications for the use of albumin should be kept in mind.

Once the patient has reached MF levels ( $1 \mathrm{~mL} / \mathrm{kg} / \mathrm{h}$ ) with an adequate UO for 2 hours, then the resuscitation can be deemed complete, and the $\mathrm{RL}$ is changed to MF containing dextrose.

Vitamin $C$ is another vital component of fluid resuscitation in burn patients, and a separate protocol has been developed to address this, 


\section{ARTICLE}

in an attempt to keep this algorithm relatively simple, teachable and easy to execute. ${ }^{[12]}$ Despite this perceived simplicity, from a specialist perspective daily challenges remain in getting staff to translate these guidelines into practice, and more education in this regard is needed, with daily monitoring of staff by those more experienced. The difficulties in teaching and perfecting fluid resuscitation are not a new phenomenon, but the solution is one we need to constantly work on. ${ }^{[11]}$

Acknowledgements. None.

Author contributions. Equal contributions.

Funding. None.

Conflicts of interest. None.
2. Guilabert P, Usua G, Martin N, et al. Fluid resuscitation management in patients with burns: Update. Br Anaesth 2016;117(3):284-296. https://doi.org/10. 1093/bja/aew266

. Bacomo FK, Chung KK. A primer on burn resuscitation. J Emerg Trauma Shock 2011:4(1):109-113. https://do org/10.4103/0974-2700.76845

4. Romanowski KS, Palmieri TL. Paediatric burn resuscitation: Past, present, and future. Burns Trauma 2017;5:26 https://doi.org/10.1186/s41038-017-0091-y

5. Saffle JR The phenomenon of 'fluid creen' in acute burn resuscitation. J Burn Care Res 2007:28(3):382-395. https://doi.org/10.1097/BCR.0B013E318053D3A1

6. Warden GD. Burn shock resuscitation. World J Surg 1992;16(1):16-23.

7. Chiao HY, Chou CY, Tzeng YS, et al. Goal-directed fluid resuscitation protocol based on arterial waveform analysis of major burn patients in a mass burn casualty. Ann Plast Surg 2018:80(Suppl 2):521- 525. https://doi arg/10.1097/SAP.0000000000001288

8. Sanchez M, Garcia-de Lorenzo A, Herrero E, et al. A protocol for resuscitation of severe burn patients guided by transpulmonary thermodilution and lactate levels: A 3-year prospective cohort study. Crit Care 2013;17(4):R176. https://doi.org/10.1186/cc12855

9. Wilms H, Mittal A, Haydock MD, et al. A systematic review of goal-directed fluid therapy: Rating of evidence for goals and monitoring. J Crit Care 2014;29(2):204-209. https://doi.org/10.1016/j.jcrc.2013.10.019

10. Kirkpatrick AW, Roberts DJ, De Waele J, et al. Intra-abdominal hypertension and the abdominal compartment syndrome: Updated consensus definitions and clinical practice guidelines from the World Society of Abdomina Compartment Syndrome. Intensive Care Med 2013;39(7):1190-1206. https://doi.org/10.1007/500134-013-2906-z 11. Hayek S, Ibrahim A, Abu Sittah G, et al. Burn resuscitation: Is it straightforward or a challenge? Ann Burn Fire Disasters 2011;24(1):17-21.

Accepted 8 November 2018
1. Haberal M, Abali AES, Karakayali H. Fluid management in major burn injuries. Indian J Plast Surg 2010;43(Suppl):S29-S36. https://doi.org/10.4103/0970-0358.70715 